\title{
On a Certain Quadratic Character Sums of Ternary Symmetry Polynomials $\bmod p$
}

\author{
Yuanyuan Meng \\ School of Mathematics, Northwest University, Xi'an, Shaanxi, China \\ Correspondence should be addressed to Yuanyuan Meng; yymeng@stumail.nwu.edu.cn
}

Received 1 March 2021; Accepted 31 March 2021; Published 17 April 2021

Academic Editor: Tingting Wang

Copyright ( $\odot 2021$ Yuanyuan Meng. This is an open access article distributed under the Creative Commons Attribution License, which permits unrestricted use, distribution, and reproduction in any medium, provided the original work is properly cited.

In this article, we are using the elementary methods and the properties of the classical Gauss sums to study the calculating problem of a certain quadratic character sums of a ternary symmetry polynomials modulo $p$ and obtain some interesting identities for them.

\section{Introduction}

Let $p$ be an odd prime, $(* / p)$ denotes the Legendre symbol $\bmod p$, i.e., for any integer $n$, one has

$$
\left(\frac{n}{p}\right)= \begin{cases}1, & \text { if } n \text { is a quadratic residue } \bmod p, \\ -1, & \text { if } n \text { is a quadratic nonresidue } \bmod p, \\ 0, & \text { if } p \mid n .\end{cases}
$$

Some of the most commonly used properties of the Legendre symbol are as follows (see $[1,2])$ :

$$
\begin{aligned}
\left(\frac{-1}{p}\right) & =(-1)^{(p-1) / 2}, \\
\left(\frac{2}{p}\right) & =(-1)^{\left(p^{2}-1\right) / 8}, \\
\left(\frac{q}{p}\right) \cdot\left(\frac{p}{q}\right) & =(-1)^{((p-1)(q-1)) / 4},
\end{aligned}
$$

where $p$ and $q$ are two different odd primes.

The introduction of the Legendre symbol has not only enriched the content of number theory but also greatly promoted the development of elementary and analytic number theory, especially the research on the properties of primes. For example, if $p$ is a prime with $p \equiv 1 \bmod 4$, then for any integers $r$ and $s$ with $(r s / p)=-1$, one has the identity (see Theorems 4-11 in [2])

$$
\begin{aligned}
p & =\alpha^{2}(p)+\beta^{2}(p) \\
& =\left(\sum_{a=1}^{(p-1) / 2}\left(\frac{a+r \bar{a}}{p}\right)\right)^{2}+\left(\sum_{b=1}^{(p-1) / 2}\left(\frac{b+s \bar{b}}{p}\right)\right)^{2} .
\end{aligned}
$$

From (3), we naturally wonder, for other forms of primes $p$, can they also be expressed in terms of Legendre's symbol?

In particular, if $p$ is an odd prime with $p \equiv 1 \bmod 3$, then there are two integers $d$ and $b$ such that the identity (see [3])

$$
4 p=d^{2}+27 b^{2}
$$

where $d$ is uniquely determined by $d \equiv 1 \bmod 3$.

In addition, if $p$ is an odd with $p \equiv 3 \bmod 4$, then there are two integers $x$ and $y$ such that

$$
p=x^{2}-2 \cdot\left(\frac{2}{p}\right) \cdot y^{2}
$$

Although we have not found the representations of $d$ and $b$ or $x$ and $y$ in terms of the Legendre symbol modulo $p$, we found that a certain quadratic character sum of the ternary symmetry polynomials are closely related to the numbers $d$ and $b$. 
In this paper, we shall use elementary methods and the properties of the classical Gauss sums to study the calculating problem of a certain quadratic character sum of binary symmetry polynomials modulo $p$ and obtain several interesting identities for them. That is, we shall prove the following results.

Theorem 1. Let $p$ be an odd prime with $(3, p-1)=1$. Then, we have

$$
\sum_{a=1}^{p-1} \sum_{b=1}^{p-1} \sum_{c=1}^{p-1}\left(\frac{a^{4} b c+b^{4} a c+c^{4} a b+a b c}{p}\right)=-\left(\frac{-1}{p}\right) \cdot p .
$$

Theorem 2. Let $p$ be an odd prime with $p \equiv 1 \bmod 6$. If 2 is a cubic residue modulo $p$, then we have the identity

$$
\begin{gathered}
\sum_{a=1}^{p-1} \sum_{b=1}^{p-1} \sum_{c=1}^{p-1}\left(\frac{a^{4} b c+b^{4} a c+c^{4} a b+a b c}{p}\right) \\
=\left(\frac{-1}{p}\right) \cdot\left(9 p d-5 p-d^{2}\right),
\end{gathered}
$$

where $d$ is the same as defined in (4).

Theorem 3. Let $p$ be an odd prime with $p \equiv 1 \operatorname{mod6}$. If 2 is not a cubic residue modulo $p$, then we have the identity

$$
\begin{aligned}
& \sum_{a=1}^{p-1} \sum_{b=1}^{p-1} \sum_{c=1}^{p-1}\left(\frac{a^{4} b c+b^{4} a c+c^{4} a b+a b c}{p}\right) \\
& =-\frac{1}{2} \cdot\left(\frac{-1}{p}\right) \cdot\left(9 p d+9 p b+10 p+2 d^{2}\right), \\
& \sum_{a=1}^{p-1} \sum_{b=1}^{p-1} \sum_{c=1}^{p-1}\left(\frac{a^{4} b c+b^{4} a c+c^{4} a b+a b c}{p}\right) \\
& =-\frac{1}{2} \cdot\left(\frac{-1}{p}\right) \cdot\left(9 p d-9 p b+10 p+2 d^{2}\right) .
\end{aligned}
$$

From these theorems, we may immediately deduce the following three corollaries.

Corollary 1. Let $p$ be an odd prime with $(3, p-1)=1$. Then, we have

$$
\left|\sum_{a=1}^{p-1} \sum_{b=1}^{p-1} \sum_{c=1}^{p-1}\left(\frac{a^{4} b c+b^{4} a c+c^{4} a b+a b c}{p}\right)\right|=p .
$$

Corollary 2. Let $p$ be a prime with $p \equiv 1 \bmod 6$. If 2 is a cubic residue modulo $p$, then we have

$$
\left|\sum_{a=1}^{p-1} \sum_{b=1}^{p-1} \sum_{c=1}^{p-1}\left(\frac{a^{4} b c+b^{4} a c+c^{4} a b+a b c}{p}\right)\right|=\left|9 p d-5 p-d^{2}\right| .
$$

Corollary 3. If $p$ is an odd prime with $p \equiv 1 \bmod 6$, if 2 is not a cubic residue modulo $p$, then we have

$$
\begin{gathered}
\left|\sum_{a=1}^{p-1} \sum_{b=1}^{p-1} \sum_{c=1}^{p-1}\left(\frac{a^{4} b c+b^{4} a c+c^{4} a b+a b c}{p}\right)\right| \\
=\frac{1}{2} \cdot\left|9 p d+9 p b+10 p+2 d^{2}\right| \text { or } \\
\frac{1}{2} \cdot\left|9 p d-9 p b+10 p+2 d^{2}\right| .
\end{gathered}
$$

Notes: it is clear that our method is applicable to multivariate symmetry polynomials $f\left(x_{1}, x_{2}, \ldots, x_{k}\right)$. But, when $k$ is larger, the calculation is more complicated, so we do not give it.

Theorem 3 is flawed. In other words, it gives us two possibilities. We do not know for sure which one is the exact value. How to determine its exact value is an open problem. Interested readers are encouraged to join us in the research.

\section{Several Lemmas}

In this section, we first give several simple lemmas. Of course, the proofs of these lemmas need some knowledge of elementary and analytic number theory. They can be found in many number theory books, such as $[1,2]$. Other papers related to Gauss sums and character sums can also be found in [4-11]; here, we do not need to list.

Lemma 1. Let $p$ be a prime with $p \equiv 1 \bmod 3$. Then, for any third-order character $\lambda \bmod p$, one has the identity

$$
\tau^{3}(\lambda)+\tau^{3}(\bar{\lambda})=d p
$$

where $d$ is the same as defined in (4).

Proof. See the work of Zhang and $\mathrm{Hu}$ [12].

Lemma 2. Let $p$ be a prime with $p \equiv 1 \operatorname{mod6}$. Then, for any sixth-order character $\psi \bmod p$, one has the identity

$$
\tau^{3}(\psi)+\tau^{3}(\bar{\psi})= \begin{cases}p^{(1 / 2)} \cdot\left(d^{2}-2 p\right), & \text { if } p \equiv 1 \bmod 12 \\ -i \cdot p^{(1 / 2)} \cdot\left(d^{2}-2 p\right), & \text { if } p \equiv 7 \bmod 12\end{cases}
$$

where $i^{2}=-1$ and $d$ is the same as defined in (4).

Proof. This result is Lemma 3 in the work of Chen [4], so we omit the proof process.

Lemma 3. Let $p$ be a prime with $p \equiv 1 \bmod 6$. Then for any third-order character $\lambda \bmod p$, we have the identity

$$
\begin{gathered}
\sum_{a=1}^{p-1} \sum_{b=1}^{p-1} \chi_{2}(a b) \sum_{c=1}^{p-1}(1+\lambda(c)+\bar{\lambda}(c)) \chi_{2}(a+b+c+1) \\
=\left(\frac{-1}{p}\right) \cdot\left(-p+\bar{\lambda}(2) \cdot \tau^{3}(\lambda)+\lambda(2) \tau^{3}(\bar{\lambda})\right),
\end{gathered}
$$

where $(* / p)=\chi_{2}$ denotes the Legendre symbol modulo $p$. 
Proof. Note that $\chi_{2}^{2}=\chi_{0}$ and $\tau^{2}\left(\chi_{2}\right)=\chi_{2}(-1) \cdot p$; from the properties of Gauss sums and the Legendre symbol modulo $p$, we have

$$
\begin{aligned}
\sum_{a=1}^{p-1} \sum_{b=1}^{p-1} \chi_{2}(a b) \sum_{c=1}^{p-1} \chi_{2}(a+b+c+1) & =-\sum_{a=1}^{p-1} \sum_{b=1}^{p-1} \chi_{2}(a b) \chi_{2}(a+b+1) \\
& =\frac{-1}{\tau\left(\chi_{2}\right)} \cdot \sum_{d=1}^{p-1} \chi_{2}(d) \sum_{a=1}^{p-1} \sum_{b=1}^{p-1} \chi_{2}(a b) e\left(\frac{d(a+b+1)}{p}\right) \\
& =-\tau\left(\chi_{2}\right) \cdot \sum_{d=1}^{p-1} \chi_{2}(d) e\left(\frac{d}{p}\right)=-\tau^{2}\left(\chi_{2}\right)
\end{aligned}
$$

It is clear that $\lambda(-1)=1$ and and from the properties of the Gauss sums, we can get

$$
\sum_{a=0}^{p-1} e\left(\frac{b a^{2}}{p}\right)=1+\sum_{a=1}^{p-1}\left(1+\left(\frac{a}{p}\right)\right) e\left(\frac{b a}{p}\right)=\chi_{2}(b) \tau\left(\chi_{2}\right)
$$

$$
\begin{aligned}
& \sum_{a=1}^{p-1} \sum_{b=1}^{p-1} \chi_{2}(a b) \sum_{c=1}^{p-1} \lambda(c) \chi_{2}(a+b+c+1) \\
& =\frac{1}{\tau\left(\chi_{2}\right)} \cdot \sum_{d=1}^{p-1} \chi_{2}(d) \sum_{a=1}^{p-1} \sum_{b=1}^{p-1} \chi_{2}(a b) \sum_{c=1}^{p-1} \lambda(c) e\left(\frac{d(a+b++c+1)}{p}\right) \\
& =\frac{\tau^{2}\left(\chi_{2}\right) \cdot \tau(\lambda)}{\tau\left(\chi_{2}\right)} \sum_{d=1}^{p-1} \chi_{2}(d) \bar{\lambda}(d) e\left(\frac{d}{p}\right)=\tau\left(\chi_{2}\right) \cdot \tau(\lambda) \cdot \tau\left(\chi_{2} \bar{\lambda}\right) \\
& \sum_{a=0}^{p-1} \chi_{2} \lambda\left(a^{2}-1\right)=\frac{1}{\tau\left(\chi_{2} \bar{\lambda}\right)} \sum_{b=1}^{p-1} \chi_{2} \bar{\lambda}(b) \sum_{a=0}^{p-1} e\left(\frac{b\left(a^{2}-1\right)}{p}\right) \\
& =\frac{\tau\left(\chi_{2}\right)}{\tau\left(\chi_{2} \bar{\lambda}\right)} \sum_{b=1}^{p-1} \chi_{2} \bar{\lambda}(b) \chi_{2}(b) e\left(\frac{-b}{p}\right)=\frac{\tau\left(\chi_{2}\right) \cdot \tau(\bar{\lambda})}{\tau\left(\chi_{2} \bar{\lambda}\right)} .
\end{aligned}
$$

On the other hand, note that $\bar{\lambda}^{2}=\lambda$; we also have

$$
\begin{aligned}
\sum_{a=0}^{p-1} \chi_{2} \lambda\left(a^{2}-1\right) & =\sum_{a=0}^{p-1} \chi_{2} \lambda\left((a+1)^{2}-1\right)=\sum_{a=1}^{p-1} \chi_{2} \lambda\left(a^{2}+2 a\right) \\
& =\frac{1}{\tau\left(\chi_{2} \bar{\lambda}\right)} \sum_{b=1}^{p-1} \chi_{2} \bar{\lambda}(b) \sum_{a=1}^{p-1} \chi_{2} \lambda(a) e\left(\frac{b(a+2)}{p}\right), \\
& =\frac{\tau\left(\chi_{2} \lambda\right)}{\tau\left(\chi_{2} \bar{\lambda}\right)} \sum_{b=1}^{p-1} \chi_{2} \bar{\lambda}(b) \chi_{2}(b) \bar{\lambda}(b) e\left(\frac{2 b}{p}\right)=\frac{\bar{\lambda}(2) \cdot \tau(\lambda) \cdot \tau\left(\chi_{2} \lambda\right)}{\tau\left(\chi_{2} \bar{\lambda}\right)}
\end{aligned}
$$


Note that $\tau(\lambda) \cdot \tau(\bar{\lambda})=p$; from (17)-(19), we have the identity

$$
\sum_{a=1}^{p-1} \sum_{b=1}^{p-1} \chi_{2}(a b) \sum_{c=1}^{p-1} \lambda(c) \chi_{2}(a+b+c+1)=\left(\frac{-1}{p}\right) \cdot \bar{\lambda}(2) \cdot \tau^{3}(\lambda) .
$$

Similarly, we also get

$\sum_{a=1}^{p-1} \sum_{b=1}^{p-1} \chi_{2}(a b) \sum_{c=1}^{p-1} \bar{\lambda}(c) \chi_{2}(a+b+c+1)=\left(\frac{-1}{p}\right) \cdot \lambda(2) \cdot \tau^{3}(\bar{\lambda})$.

Now, combining (15), (20), and (21), we have the identity

$$
\begin{aligned}
\sum_{a=1}^{p-1} & \sum_{b=1}^{p-1} \chi_{2}(a b) \sum_{c=1}^{p-1}(1+\lambda(c)+\bar{\lambda}(c)) \chi_{2}(a+b+c+1) \\
& =-\left(\frac{-1}{p}\right) \cdot p+\left(\frac{-1}{p}\right) \cdot\left(\bar{\lambda}(2) \cdot \tau^{3}(\lambda)+\lambda(2) \tau^{3}(\bar{\lambda})\right) .
\end{aligned}
$$

This proves Lemma 3.

Lemma 4. Let $p$ be a prime with $p \equiv 1 \operatorname{mod6}$. Then, for any third-order character $\lambda \bmod p$, we have the identity

$$
\begin{gathered}
\sum_{a=1}^{p-1} \sum_{b=1}^{p-1} \chi_{2}(a b) \lambda(a) \sum_{c=1}^{p-1}(1+\lambda(c)+\bar{\lambda}(c)) \chi_{2}(a+b+c+1) \\
=-\left(\frac{-1}{p}\right) \cdot p+\left(\frac{-1}{p}\right) \cdot(\lambda(2)+\bar{\lambda}(2)) \cdot \tau^{3}(\bar{\lambda}), \\
\sum_{a=1}^{p-1} \sum_{b=1}^{p-1} \chi_{2}(a b) \bar{\lambda}(a) \sum_{c=1}^{p-1}(1+\lambda(c)+\bar{\lambda}(c)) \chi_{2}(a+b+c+1) \\
=-\left(\frac{-1}{p}\right) \cdot p+\left(\frac{-1}{p}\right) \cdot(\lambda(2)+\bar{\lambda}(2)) \cdot \tau^{3}(\bar{\lambda}) .
\end{gathered}
$$

Proof. Note that $\tau\left(\chi_{2} \lambda\right) \cdot \tau\left(\chi_{2} \bar{\lambda}\right)=\chi_{2}(-1) \cdot p$; from the methods of proving Lemma 3 , we have

$$
\begin{aligned}
& \sum_{a=1}^{p-1} \sum_{b=1}^{p-1} \chi_{2}(a b) \lambda(a) \sum_{c=1}^{p-1} \chi_{2}(a+b+c+1) \\
& =-\sum_{a=1}^{p-1} \sum_{b=1}^{p-1} \chi_{2}(a b) \lambda(a) \chi_{2}(a+b+1) \\
& =-\frac{1}{\tau\left(\chi_{2}\right)} \sum_{c=1}^{p-1} \chi_{2}(c) \sum_{a=1}^{p-1} \sum_{b=1}^{p-1} \chi_{2}(a b) \lambda(a) e\left(\frac{c(a+b+1)}{p}\right) \\
& =-\tau\left(\chi_{2} \lambda\right) \cdot \tau\left(\chi_{2} \bar{\lambda}\right)=-\left(\frac{-1}{p}\right) \cdot p .
\end{aligned}
$$

$$
\begin{gathered}
\sum_{a=1}^{p-1} \sum_{b=1}^{p-1} \chi_{2}(a b) \lambda(a) \sum_{c=1}^{p-1} \lambda(c) \chi_{2}(a+b+c+1) \\
=\frac{1}{\tau\left(\chi_{2}\right)} \sum_{d=1}^{p-1} \chi_{2}(d) \sum_{a=1}^{p-1} \sum_{b=1}^{p-1} \chi_{2}(a b) \lambda(a)
\end{gathered}
$$$$
\cdot \sum_{c=1}^{p-1} \lambda(c) e\left(\frac{d(a+b++c+1)}{p}\right)
$$

$$
\begin{aligned}
& =\tau(\lambda) \cdot \tau\left(\chi_{2} \lambda\right) \cdot \sum_{d=1}^{p-1} \chi_{2}(d) \lambda(d) e\left(\frac{d}{p}\right) \\
& =\tau(\lambda) \cdot \tau^{2}\left(\chi_{2} \lambda\right), \\
& =\left(\frac{-1}{p}\right) \cdot \bar{\lambda}(2) \cdot \tau^{3}(\bar{\lambda}) .
\end{aligned}
$$

Similarly, we also have

$$
\begin{aligned}
& \sum_{a=1}^{p-1} \sum_{b=1}^{p-1} \chi_{2}(a b) \lambda(a) \sum_{c=1}^{p-1} \bar{\lambda}(c) \chi_{2}(a+b+c+1) \\
& =\left(\frac{-1}{p}\right) \cdot \lambda(2) \cdot \tau^{3}(\bar{\lambda}) .
\end{aligned}
$$

Combining (24)-(26), we have

$$
\begin{aligned}
& \sum_{a=1}^{p-1} \sum_{b=1}^{p-1} \chi_{2}(a b) \lambda(a) \sum_{c=1}^{p-1}(1+\lambda(c)+\bar{\lambda}(c)) \chi_{2}(a+b+c+1) \\
& =-\left(\frac{-1}{p}\right) \cdot p+\left(\frac{-1}{p}\right) \cdot(\lambda(2)+\bar{\lambda}(2)) \cdot \tau^{3}(\bar{\lambda}) .
\end{aligned}
$$

Taking the conjugate in (27), we have

$$
\begin{array}{r}
\sum_{a=1}^{p-1} \sum_{b=1}^{p-1} \chi_{2}(a b) \bar{\lambda}(a) \sum_{c=1}^{p-1}(1+\lambda(c)+\bar{\lambda}(c)) \chi_{2}(a+b+c+1) \\
=-\left(\frac{-1}{p}\right) \cdot p+\left(\frac{-1}{p}\right) \cdot(\lambda(2)+\bar{\lambda}(2)) \cdot \tau^{3}(\lambda) .
\end{array}
$$

Now, Lemma 4 follows from (27) and (28).

Lemma 5. Let $p$ be a prime with $p \equiv 1 \operatorname{mod6}$. Then, for any third-order character $\lambda \bmod p$, we have the identities 


$$
\begin{gathered}
\sum_{a=1}^{p-1} \sum_{b=1}^{p-1} \chi_{2}(a b) \lambda(a b) \sum_{c=1}^{p-1}(1+\lambda(c)+\bar{\lambda}(c)) \chi_{2}(a+b+c+1) \\
=-\frac{\tau^{3}\left(\chi_{2} \lambda\right)}{\tau\left(\chi_{2}\right)}+\left(\frac{-1}{p}\right) \cdot\left(\bar{\lambda}(2) \cdot \tau^{3}(\bar{\lambda})+\lambda(2) \cdot \tau^{3}(\lambda)\right), \\
\sum_{a=1}^{p-1} \sum_{b=1}^{p-1} \chi_{2}(a b) \bar{\lambda}(a b) \sum_{c=1}^{p-1}(1+\lambda(c)+\bar{\lambda}(c)) \chi_{2}(a+b+c+1) \\
=-\frac{\tau^{3}\left(\chi_{2} \lambda\right)}{\tau\left(\chi_{2}\right)}+\left(\frac{-1}{p}\right) \cdot\left(\bar{\lambda}(2) \cdot \tau^{3}(\bar{\lambda})+\lambda(2) \cdot \tau^{3}(\lambda)\right) .
\end{gathered}
$$

Proof. Note that $\bar{\lambda}^{2}=\lambda$; from (18), (19), and the methods of proving Lemma 3, we have

$$
\begin{aligned}
& \sum_{a=1}^{p-1} \sum_{b=1}^{p-1} \chi_{2}(a b) \lambda(a b) \sum_{c=1}^{p-1} \chi_{2}(a+b+c+1) \\
& \quad=-\sum_{a=1}^{p-1} \sum_{b=1}^{p-1} \chi_{2}(a b) \lambda(a b) \chi_{2}(a+b+1) \\
& \quad=-\frac{1}{\tau\left(\chi_{2}\right)} \sum_{c=1}^{p-1} \chi_{2}(c) \sum_{a=1}^{p-1} \sum_{b=1}^{p-1} \chi_{2}(a b) \lambda(a b) e\left(\frac{c(a+b+1)}{p}\right)=-\frac{\tau^{3}\left(\chi_{2} \lambda\right)}{\tau\left(\chi_{2}\right)} \\
& \sum_{a=1}^{p-1} \sum_{b=1}^{p-1} \chi_{2}(a b) \lambda(a b) \sum_{c=1}^{p-1} \lambda(c) \chi_{2}(a+b+c+1) \\
& =\frac{1}{\tau\left(\chi_{2}\right)} \sum_{d=1}^{p-1} \chi_{2}(d) \sum_{a=1}^{p-1} \sum_{b=1}^{p-1} \chi_{2}(a b) \lambda(a b) \sum_{c=1}^{p-1} \lambda(c) e\left(\frac{d(a+b++c+1)}{p}\right) \\
& \quad=\tau(\lambda) \cdot \tau^{2}\left(\chi_{2} \lambda\right)=\left(\frac{-1}{p}\right) \cdot \bar{\lambda}(2) \cdot \tau^{3}(\bar{\lambda}) \\
& \sum_{a=1}^{p-1} \sum_{b=1}^{p-1} \chi_{2}(a b) \lambda(a b) \sum_{c=1}^{p-1} \bar{\lambda}(c) \chi_{2}(a+b+c+1) \\
& \quad=\frac{1}{\tau\left(\chi_{2}\right)} \sum_{d=1}^{p-1} \chi_{2}(d) \sum_{a=1}^{p-1} \sum_{b=1}^{p-1} \chi_{2}(a b) \lambda(a b) \sum_{c=1}^{p-1} \bar{\lambda}(c) e\left(\frac{d(a+b++c+1)}{p}\right) \\
& \quad=\frac{1}{\tau\left(\chi_{2}\right)} \cdot \tau(\bar{\lambda}) \cdot \tau^{2}\left(\chi_{2} \lambda\right) \cdot \tau\left(\chi_{2} \bar{\lambda}\right)=\left(\frac{-1}{p}\right) \cdot \lambda(2) \cdot \tau^{3}(\lambda) .
\end{aligned}
$$

Combining (30)-(32), we have

$$
\begin{gathered}
\sum_{a=1}^{p-1} \sum_{b=1}^{p-1} \chi_{2}(a b) \lambda(a b) \sum_{c=1}^{p-1}(1+\lambda(c)+\bar{\lambda}(c)) \chi_{2}(a+b+c+1) \\
=-\frac{\tau^{3}\left(\chi_{2} \lambda\right)}{\tau\left(\chi_{2}\right)}+\left(\frac{-1}{p}\right) \cdot\left(\bar{\lambda}(2) \cdot \tau^{3}(\bar{\lambda})+\lambda(2) \cdot \tau^{3}(\lambda)\right) .
\end{gathered}
$$


Taking the conjugate given above, we can deduce the other identity. This proves Lemma 5.
Lemma 6. Let $p$ be a prime with $p \equiv 1 \bmod 6$. Then, for any third-order character $\lambda \bmod p$, we have the identity

$$
\begin{gathered}
\sum_{a=1}^{p-1} \sum_{b=1}^{p-1} \chi_{2}(a b) \lambda(a) \bar{\lambda}(b) \sum_{c=1}^{p-1}(1+\lambda(c)+\bar{\lambda}(c)) \chi_{2}(a+b+c+1) \\
=-\left(\frac{-1}{p}\right) \cdot p+\left(\frac{-1}{p}\right) \cdot\left(\lambda(2) \cdot \tau^{3}(\bar{\lambda})+\bar{\lambda}(2) \cdot \tau^{3}(\lambda)\right) .
\end{gathered}
$$

Proof. From (18), (19), and the methods of proving Lemma

3 , we have

$$
\begin{aligned}
& \sum_{a=1}^{p-1} \sum_{b=1}^{p-1} \chi_{2}(a b) \lambda(a) \bar{\lambda}(b) \sum_{c=1}^{p-1} \chi_{2}(a+b+c+1) \\
& =-\sum_{a=1}^{p-1} \sum_{b=1}^{p-1} \chi_{2}(a b) \lambda(a) \bar{\lambda}(b) \chi_{2}(a+b+1) \\
& =-\frac{1}{\tau\left(\chi_{2}\right)} \sum_{c=1}^{p-1} \chi_{2}(c) \sum_{a=1}^{p-1} \sum_{b=1}^{p-1} \chi_{2}(a b) \lambda(a) \bar{\lambda}(b) e\left(\frac{c(a+b+1)}{p}\right), \\
& =-\tau\left(\chi_{2} \lambda\right) \tau\left(\chi_{2} \bar{\lambda}\right)=-\left(\frac{-1}{p}\right) \cdot p \text {, } \\
& \sum_{a=1}^{p-1} \sum_{b=1}^{p-1} \chi_{2}(a b) \lambda(a) \bar{\lambda}(b) \sum_{c=1}^{p-1} \lambda(c) \chi_{2}(a+b+c+1) \\
& =\frac{1}{\tau\left(\chi_{2}\right)} \sum_{d=1}^{p-1} \chi_{2}(d) \sum_{a=1}^{p-1} \sum_{b=1}^{p-1} \chi_{2}(a b) \lambda(a) \bar{\lambda}(b) \sum_{c=1}^{p-1} \lambda(c) e\left(\frac{d(a+b++c+1)}{p}\right), \\
& =\frac{\tau(\lambda) \cdot \tau^{2}\left(\chi_{2} \bar{\lambda}\right) \cdot \tau\left(\chi_{2} \lambda\right)}{\tau\left(\chi_{2}\right)}=\left(\frac{-1}{p}\right) \cdot \bar{\lambda}(2) \cdot \tau^{3}(\lambda), \\
& \sum_{a=1}^{p-1} \sum_{b=1}^{p-1} \chi_{2}(a b) \lambda(a) \bar{\lambda}(b) \sum_{c=1}^{p-1} \bar{\lambda}(c) \chi_{2}(a+b+c+1) \\
& =\frac{1}{\tau\left(\chi_{2}\right)} \sum_{d=1}^{p-1} \chi_{2}(d) \sum_{a=1}^{p-1} \sum_{b=1}^{p-1} \chi_{2}(a b) \lambda(a) \bar{\lambda}(b) \sum_{c=1}^{p-1} \bar{\lambda}(c) e\left(\frac{d(a+b++c+1)}{p}\right), \\
& =\frac{\tau(\lambda) \cdot \tau^{2}\left(\chi_{2} \bar{\lambda}\right) \cdot \tau\left(\chi_{2} \lambda\right)}{\tau\left(\chi_{2}\right)}=\left(\frac{-1}{p}\right) \cdot \lambda(2) \cdot \tau^{3}(\lambda) .
\end{aligned}
$$

Combining (35)-(37), we have

$$
\begin{aligned}
& \sum_{a=1}^{p-1} \sum_{b=1}^{p-1} \chi_{2}(a b) \lambda(a) \bar{\lambda}(b) \sum_{c=1}^{p-1}(1+\lambda(c)+\bar{\lambda}(c)) \chi_{2}(a+b+c+1) \\
& =-\left(\frac{-1}{p}\right) \cdot p+\left(\frac{-1}{p}\right) \cdot\left(\lambda(2) \cdot \tau^{3}(\bar{\lambda})+\bar{\lambda}(2) \cdot \tau^{3}(\lambda)\right) .
\end{aligned}
$$

This proves Lemma 6. 


\section{Proofs of the Theorems}

Now, we prove our theorems. From the properties of the reduced residue system modulo $p$, we have

$$
\begin{aligned}
\sum_{a=1}^{p-1} & \sum_{b=1}^{p-1} \sum_{c=1}^{p-1} \chi_{2}\left(a^{4} b c+b^{4} a c+c^{4} a b+a b c\right) \\
& =\sum_{a=1}^{p-1} \sum_{b=1}^{p-1} \sum_{c=1}^{p-1} \chi_{2}\left(a^{4} b c^{6}+b^{4} a c^{6}+c^{6} a b+a b c^{3}\right), \\
& =\sum_{a=1}^{p-1} \sum_{b=1}^{p-1} \chi_{2}(a b) \sum_{c=1}^{p-1} \chi_{2}\left(a^{3}+b^{3}+1+\bar{c}^{3}\right), \\
& =\sum_{a=1}^{p-1} \sum_{b=1}^{p-1} \chi_{2}\left(a^{3} b^{3}\right) \sum_{c=1}^{p-1} \chi_{2}\left(a^{3}+b^{3}+c^{3}+1\right) .
\end{aligned}
$$

If $(3, p-1)=1$, when $a$ passes through a reduced residue system modulo $p$, then $a^{3}$ also passes through a reduced residue system modulo $p$. So, from (39) and (15), we have the identity

$$
\begin{aligned}
& \sum_{a=1}^{p-1} \sum_{b=1}^{p-1} \sum_{c=1}^{p-1} \chi_{2}\left(a^{4} b c+b^{4} a c+c^{4} a b+a b c\right) \\
& \quad=\sum_{a=1}^{p-1} \sum_{b=1}^{p-1} \chi_{2}(a b) \sum_{c=1}^{p-1} \chi_{2}(a+b+c+1)=-\tau^{2}\left(\chi_{2}\right) \\
& =-\left(\frac{-1}{p}\right) \cdot p .
\end{aligned}
$$

This proves Theorem 1 .

If $p \equiv 1 \bmod 6$, let $\lambda$ be a third-order character modulo $p$; then, for any integer $a$ with $(a, p)=1$, we have the identity

$$
1+\lambda(a)+\bar{\lambda}(a)= \begin{cases}3, & \text { if } a \text { is a cubic residue modulo } p ; \\ 0, & \text { if } a \text { is not a cubic residue modulo } p .\end{cases}
$$

So, from the symmetry of $a$ and $b$, (39), and Lemmas 3-6, we have

$$
\begin{aligned}
& \sum_{a=1}^{p-1} \sum_{b=1}^{p-1} \sum_{c=1}^{p-1} \chi_{2}\left(a^{4} b c+b^{4} a c+c^{4} a b+a b c\right) \\
&=\sum_{a=1}^{p-1} \sum_{b=1}^{p-1} \chi_{2}(a) \chi_{2}(b)(1+\lambda(a)+\bar{\lambda}(a)) \cdot(1+\lambda(b)+\bar{\lambda}(b)) \\
& \times \sum_{c=1}^{p-1}(1+\lambda(c)+\bar{\lambda}(c)) \chi_{2}(a+b+c+1) \\
&= \sum_{a=1}^{p-1} \sum_{b=1}^{p-1} \chi_{2}(a) \chi_{2}(b)(1+2 \lambda(a)+2 \bar{\lambda}(a)+\lambda(a b)+\bar{\lambda}(a b)+2 \lambda(a) \bar{\lambda}(b)) \\
& \times \sum_{c=1}^{p-1}(1+\lambda(c)+\bar{\lambda}(c)) \chi_{2}(a+b+c+1) \\
&=\left(\frac{-1}{p}\right) \cdot\left(-p+\bar{\lambda}(2) \cdot \tau^{3}(\lambda)+\lambda(2) \tau^{3}(\bar{\lambda})\right) \\
&=-7\left(\frac{-1}{p}\right) \cdot p+\left(\frac{-1}{p}\right) \cdot\left((4 \lambda(2)+5 \bar{\lambda}(2)) \cdot \tau^{3}(\lambda)+(5 \lambda(2)+4 \bar{\lambda}(2)) \tau^{3}(\bar{\lambda})\right) \\
&-2\left(\frac{-1}{p}\right) \cdot p+2\left(\frac{-1}{p}\right)(\bar{\lambda}(2)+\lambda(2)) \cdot \tau^{3}(\bar{\lambda}) \\
&-2\left(\frac{-1}{p}\right) \cdot p+2\left(\frac{-1}{p}\right) \cdot\left(\lambda(2) \cdot \tau^{3}(\bar{\lambda})+\bar{\lambda}(2) \cdot \tau^{3}(\lambda)\right) \\
&-\frac{\tau^{3}\left(\chi_{2} \bar{\lambda}\right)}{\tau\left(\chi_{2}\right)}+\left(\frac{-1}{p}\right) \cdot\left(\bar{\lambda}(2) \cdot \tau^{3}(\bar{\lambda})+\lambda(2) \cdot \tau^{3}(\lambda)\right) \\
&-\frac{\tau^{3}\left(\chi_{2} \lambda\right)}{\tau\left(\chi_{2}\right)}+\left(\frac{-1}{p}\right) \cdot\left(\bar{\lambda}(2) \tau^{3}(\bar{\lambda})+\lambda(2) \cdot \tau^{3}(\lambda)\right) \\
& p(\bar{\lambda}(2)+\lambda(2)) \cdot \tau^{3}(\lambda)
\end{aligned}
$$


If 2 is a cubic residue modulo $p$, then $\lambda(2)=\bar{\lambda}(2)=1$. Note that $\chi_{2} \lambda=\psi$ is a sixth-order character modulo $p$, $\chi_{2}(-1)=1$, and $\tau\left(\chi_{2}\right)=\sqrt{p}$, if $p \equiv 1 \bmod 12 ; \chi_{2}(-1)=-1$ and $\tau\left(\chi_{2}\right)=i \cdot \sqrt{p}$, if $p \equiv 7 \bmod 12$. From (42) and Lemmas 1 and 2 , we have

$$
\begin{gathered}
\sum_{a=1}^{p-1} \sum_{b=1}^{p-1} \sum_{c=1}^{p-1}\left(\frac{a^{4} b c+b^{4} a c+c^{4} a b+a b c}{p}\right) \\
=\left(\frac{-1}{p}\right) \cdot\left(9 p d-5 p-d^{2}\right) .
\end{gathered}
$$

This proves Theorem 2 .

Now, we prove Theorem 3 . If 2 is not a cubic residue modulo $p$, then we have $1+\lambda(2)+\bar{\lambda}(2)=0$ or $\lambda(2)+\bar{\lambda}(2)=-1$. From (42) and Lemmas 1 and 2 , we have

$$
\begin{aligned}
\sum_{a=1}^{p-1} \sum_{b=1}^{p-1} \sum_{c=1}^{p-1}\left(\frac{a^{4} b c+b^{4} a c+c^{4} a b+a b c}{p}\right) & \\
= & -\left(\frac{-1}{p}\right) \cdot\left(4 p d+5 p+d^{2}\right) \\
& +\left(\frac{-1}{p}\right) \cdot\left(\bar{\lambda}(2) \cdot \tau^{3}(\lambda)+\lambda(2) \tau^{3}(\bar{\lambda})\right) .
\end{aligned}
$$
have

Since $1+\lambda(2)+\bar{\lambda}(2)=0$ or $\lambda^{2}(2)+\lambda(2)+1=0$, we

$$
\begin{aligned}
\lambda(2) & =-\frac{1}{2} \pm \frac{\sqrt{3}}{2} \cdot i \\
\bar{\lambda}(2) & =-\frac{1}{2} \mp \frac{\sqrt{3}}{2} \cdot i, \quad \text { where } i^{2}=-1, \\
\left(\tau^{3}(\lambda)-\tau^{3}(\bar{\lambda})\right)^{2} & =\left(\tau^{3}(\lambda)+\tau^{3}(\bar{\lambda})\right)^{2}-4 p^{3}, \\
& =(\mathrm{d} p)^{2}-4 p^{3}=p^{2}\left(d^{2}-4 p\right)=-27 p^{2} b^{2},
\end{aligned}
$$

or

$$
\tau^{3}(\lambda)-\tau^{3}(\bar{\lambda})= \pm 3 \cdot \sqrt{3} \cdot p \cdot b \cdot i
$$

So, from (45)-(47) and Lemma 1, we have

$$
\bar{\lambda}(2) \cdot \tau^{3}(\lambda)+\lambda(2) \tau^{3}(\bar{\lambda})=-\frac{d p}{2} \pm \frac{9 p b}{2} .
$$

Combining (44) and (48), we have the identity

$$
\begin{aligned}
& \sum_{a=1}^{p-1} \sum_{b=1}^{p-1} \sum_{c=1}^{p-1}\left(\frac{a^{4} b c+b^{4} a c+c^{4} a b+a b c}{p}\right) \\
& \quad=-\left(\frac{-1}{p}\right) \cdot\left(4 p d+5 p+d^{2}\right)-\left(\frac{-1}{p}\right) \cdot \frac{p}{2} \cdot(d \pm 9 b) .
\end{aligned}
$$

This completes the proof of Theorem 3.

\section{Conclusions}

The main results of this paper are three identities involving a certain quadratic character sums of ternary symmetry polynomials modulo $p$. Theorem 1 proved an exact identity for the sum, if $(3, p-1)=1$. Theorem 2 discussed the case $p \equiv 1 \bmod 3$ and obtained an exact identity, provided 2 is a cubic residue modulo $p$. Theorem 3 also discussed the case $p \equiv 1 \bmod 3$, where 2 is not a cubic nonresidue modulo $p$. In this case, two possibilities are given. These results not only give the exact values of a certain quadratic character sums of ternary polynomials modulo $p$ but also some new contribution to research in related fields.

\section{Data Availability}

No data were used in this paper.

\section{Conflicts of Interest}

The authors declare that there are no conflicts of interest regarding the publication of this paper.

\section{Acknowledgments}

This work was supported by the N. S. F. (11771351) of P. R. China.

\section{References}

[1] T. M. Apostol, Introduction to Analytic Number Theory, Springer-Verlag, New York, NY, USA, 1976.

[2] W. P. Zhang and H. L. Li, Elementary Number Theory, Shaanxi Normal University Press, Xi'an, China, 2008.

[3] S. Chowla, J. Cowles, and M. Cowles, "On the number of zeros of diagonal cubic forms," Journal of Number Theory, vol. 9, no. 4, pp. 502-506, 1977.

[4] L. Chen, "On classical Gauss sums and some of their properties," Symmetry, vol. 10, no. 11, p. 625, 2018.

[5] Z. Y. Chen and W. P. Zhang, "On the fourth-order linear recurrence formula related to classical Gauss sums," Open Mathematics, vol. 15, pp. 1251-1255, 2017.

[6] L. Chen and Z. Y. Chen, "Some new hybrid power mean formulae of trigonometric sums," Advances in Differences Equation, vol. 2020, p. 220, 2020.

[7] L. Chen and J. Y. Hu, "A linear recurrence formula involving cubic Gauss sums and kloosterman sums," Acta Mathematica Sinica (Chinese Series), vol. 61, pp. 67-72, 2018.

[8] B. C. Berndt and R. J. Evans, "The determination of Gauss sums," Bulletin of the American Mathematical Society, vol. 5, no. 2, pp. 107-130, 1981

[9] W. P. Zhang and Y. Yi, "On dirichlet characters of polynomials," Bulletin of the London Mathematical Society, vol. 34, pp. 469-473, 2002.

[10] W. Zhang and W. Yao, "A note on the dirichlet characters of polynomials," Acta Arithmetica, vol. 115, no. 3, pp. 225-229, 2004.

[11] S. M. Shen and W. P. Zhang, "On the quartic Gauss sums and their recurrence property," Advances in Difference Equations, vol. 2017, p. 43, 2017.

[12] W. P. Zhang and J. Y. Hu, "The number of solutions of the diagonal cubic congruence equation $\bmod p$," Mathematical Reports, vol. 20, pp. 70-76, 2018. 